\title{
Capsaicin induces apoptosis and modulates MAPK signaling in human gastric cancer cells
}

\author{
SEON-YOUNG PARK, JI-YOUNG KIM, SU-MI LEE, CHUNG-HWAN JUN, SUNG-BUM CHO, \\ CHANG-HWAN PARK, YOUNG-EUN JOO, HYUN-SOO KIM, SUNG-KYU CHOI and JONG-SUN REW \\ Department of Internal Medicine, Chonnam National University Hospital, \\ Dong-ku, Gwangju 501-757, Republic of Korea
}

Received July 28, 2013; Accepted December 3, 2013

DOI: $10.3892 / \mathrm{mmr} .2013 .1849$

\begin{abstract}
Capsaicin is known to have tumor suppressive effects. However, the molecular mechanisms and targets of capsaicin involved in exerting anticancer activity are complex and remain to be clarified. The aim of the current study was to investigate the effects of capsaicin on human gastric cancer cells (AGS cells) and demonstrate that capsaicin induced apoptosis in AGS cells. Results of the MTT assay and flow cytometry revealed that capsaicin potentially inhibited the proliferation of AGS cells and induced apoptosis in vitro in a dose-dependent manner. Cleaved caspase-3 was increased and $\mathrm{Bcl}-2$ was reduced by treatment with capsaicin in AGS cells. Capsaicin treatment decreased the expression of phosphorylated ERK 1/2, p38 MAPK or JNK in AGS cells. The results of this study suggest that capsaicin may serve as an anti-tumorigenic agent in human gastric cancer.
\end{abstract}

\section{Introduction}

Capsaicin (trans-8-methyl-N-vanillyl-6-nonenamide), a vanilloid receptor agonist, is the principal pungent constituent of the plant genus Capsicum that is present in chilli peppers (1). The relationship of capsaicin to genotoxicity, carcinogenicity, and prevention and treatment of cancer has been widely explored (2). This alkaloid compound has been found to inhibit cancer growth and progression in vitro and in vivo and to induce apoptosis in various types of cancer cells (3-8). However, several investigations of the carcinogenic potential of capsaicin in animal models have also been reported $(2,8,9)$. The molecular mechanisms underlying capsaicin-induced apoptosis are cell-type dependent. Despite the cumulative evidence for the tumor suppressive effects of capsaicin, few studies have been undertaken concerning the effects of

Correspondence to: Professor Jong-Sun Rew, Department of Internal Medicine, Chonnam National University Medical School, 8 Hak-dong, Dong-ku, Gwangju 501-757, Republic of Korea E-mail: drpsy@jnu.ac.kr

Key words: capsaicin, apoptosis, human gastric cancer cells capsaicin on cell signaling and molecular pathways leading to apoptosis in gastric cancer (10-12).

In the present study, we investigated the effects of capsaicin on human gastric cancer cells and demonstrated that capsaicin induced apoptosis and autophagy in human gastric cancer cells.

\section{Materials and methods}

Cell cultures and culture condition. Human gastric carcinoma AGS cells were purchased from the American Type Culture collection (Manassas, VA, USA). The cells were cultured at $37^{\circ} \mathrm{C}$ in a $5 \% \mathrm{CO}_{2}$ atmosphere with Dulbecco's modified Eagle's medium supplemented with $10 \%$ fetal bovine serum and $1 \%$ penicillin-streptomycin. Capsaicin was purchased from Sigma Chemical Co. (St. Louis, MO, USA) and prepared as an ethanol stock solution, which was then diluted in intracellular solution prior to use.

Measurement of cell proliferation. Cell viability was determined using the 3-(4,5-dimethylthiazole-2-yl)-2,5diphenyltetrazolium bromide assay. Briefly, $5 \times 10^{5}$ cells/well were seeded in 96-well plates and allowed to adhere overnight. The concentrations of capsaicin used in this experiment were 0 , $10,20,30,60,80,100,200$ and $300 \mu \mathrm{M}$. Each group contained three wells. After the samples were treated with capsaicin for $12 \mathrm{~h}, 10 \mu \mathrm{l}$ of MTT $(0.5 \mathrm{mg} / \mathrm{ml}$; Sigma) was added to each well, and the cells were incubated at $37^{\circ} \mathrm{C}$ for $4 \mathrm{~h}$. The reaction was then stopped by lysing the cells with $200 \mu \mathrm{l}$ of dimethyl sulfoxide (DMSO) for 15 min. Quantification measurements (optical density) were obtained at a wavelength of $450 \mathrm{~nm}$ using spectrophotometric analysis.

Effect of capsaicin on apoptosis of AGS cells as determined by flow cytometry. To evaluate the impact of capsaicin on apoptosis, FACS analysis was performed. Cell apoptosis was assessed using the Annexin V assay (Annexin V-FITC apoptosis detection kit; BD Pharmingen, San Jose, CA, USA). AGS cells ( $1 \times 10^{5}$ cells) were treated with various concentrations $(0-300 \mu \mathrm{M})$ of capsaicin for $12 \mathrm{~h}$, respectively. Annexin V-FITC and propidium iodide (PI) labeling was then performed according to the manufacturer's instructions. The cells were double-stained with Annexin V-fluorescein isothiocyanate 
(FITC) and PI, followed by flow cytometry. To quantify early apoptotic cells, flow cytometry was used to assess binding of fluorescence-labeled Annexin V to externalized phosphatidylserine. PI uptake was measured to assess cells in the late stages of apoptosis or cells that sustained direct plasma membrane damage.

Western blotting. Total protein was lysed with RIFA buffer (1 M Tris-HCl, $150 \mathrm{mM} \mathrm{NaCl}, 1 \%$ Triton X-100, $2 \mathrm{mM}$ EDTA) with $1 \mathrm{mM}$ phenylmethanesulfonyl fluoride (PMSF) and Halt ${ }^{\mathrm{TM}}$ protease inhibitor cocktail (Thermo Scientific, Rockford, IL, USA). Protein concentrations of the cell lysate were quantified using $\mathrm{BCA}^{\mathrm{TM}}$ protein assay (Thermo Scientific) with bovine serum albumin (BSA) as a standard. Total protein $(10-20 \mu \mathrm{g})$ was subjected to electrophoresis on $10 \%$ SDS-polyacrylamide gels and then transferred to a polyvinylidene fluoride membrane (Millipore, Billerica, MA, USA). The membrane was incubated for $1 \mathrm{~h}$ in blocking solution (5\% BSA in TBS-Tween 20 buffer) and sequentially blotted with primary antibodies at $4^{\circ} \mathrm{C}$ overnight. Antibodies against c-Jun N-terminal kinase (JNK), phospho-JNK, extracellular signalregulated kinase 1/2 (ERK 1/2), phospho-ERK 1/2 (1:1,000 dilution; Cell Signaling Technology, Inc., Danvers, MA, USA), p38 mitogen-activated protein kinase (MAPK), phospho-p38 MAPK, caspase 3, cleaved caspase 3, Bcl-2, and $\beta$-actin were purchased from Cell Signaling Technology (Beverly, MA, USA). After rinsing in TST, the membrane was incubated with horseradish peroxidase (HRP)-labeled anti-rabbit or antimouse immunoglobulins as the secondary antibody (1:5,000 dilution, Santa Cruz Biotechnology, Inc., Santa Cruz, USA) at room temperature for $1 \mathrm{~h}$. Enhanced chemiluminescence was used to visualize the bands by LAS 3000 (Fuji Film, Tokyo, Japan).

Statistical analysis. The Student's t-test was used to determine the significance of differences between treatments and respective controls. Values are expressed as means $\pm \mathrm{SE}$ from at least three independent experiments. $\mathrm{P}<0.05$ was considered to indicate statistical significance. Statistical analysis was assessed using Statistical Package for the Social Sciences (SPSS/PC 20.0, Chicago, IL, USA).

\section{Results}

Capsaicin suppresses proliferation in human gastric cancer cells. An MTT assay was used to assess the potential effects of capsaicin on the proliferation of human gastric cancer cells that had been exposed to capsaicin for $12 \mathrm{~h}$ at different concentrations. After treatment with capsaicin at concentrations ranging from 10 to $300 \mu \mathrm{M}$ for $12 \mathrm{~h}$, the cell proliferation was significantly lower than that of the untreated corresponding control groups $(\mathrm{P}<0.001)$. Capsaicin decreased the proliferation of the gastric cancer cell line in vitro in a dose-dependent manner (Fig. 1).

Capsaicin induces apoptosis in human gastric cancer cells. Regulation of apoptosis is crucial for the preservation of homeostasis and morphogenesis of human tissue (3). Disturbance of these processes by aberrantly extending cell viability or favoring accumulation of transforming mutation

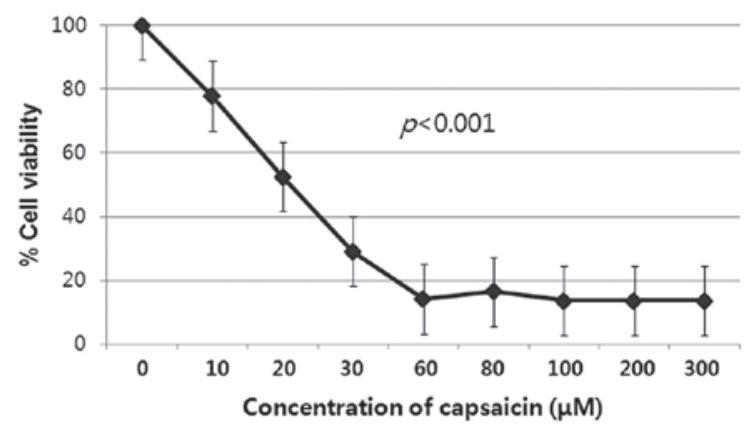

Figure 1. Capsaicin inhibits the proliferation of human gastric cancer cells Cells were plated at a density of $5 \times 10^{3}$ cells/well (three replicates/group) in 96-well plates and exposed to capsaicin treatment for $12 \mathrm{~h}$. Cell viability was determined by MTT assay and indicated as a percentage of the control. The result are representative of three independent experiments.

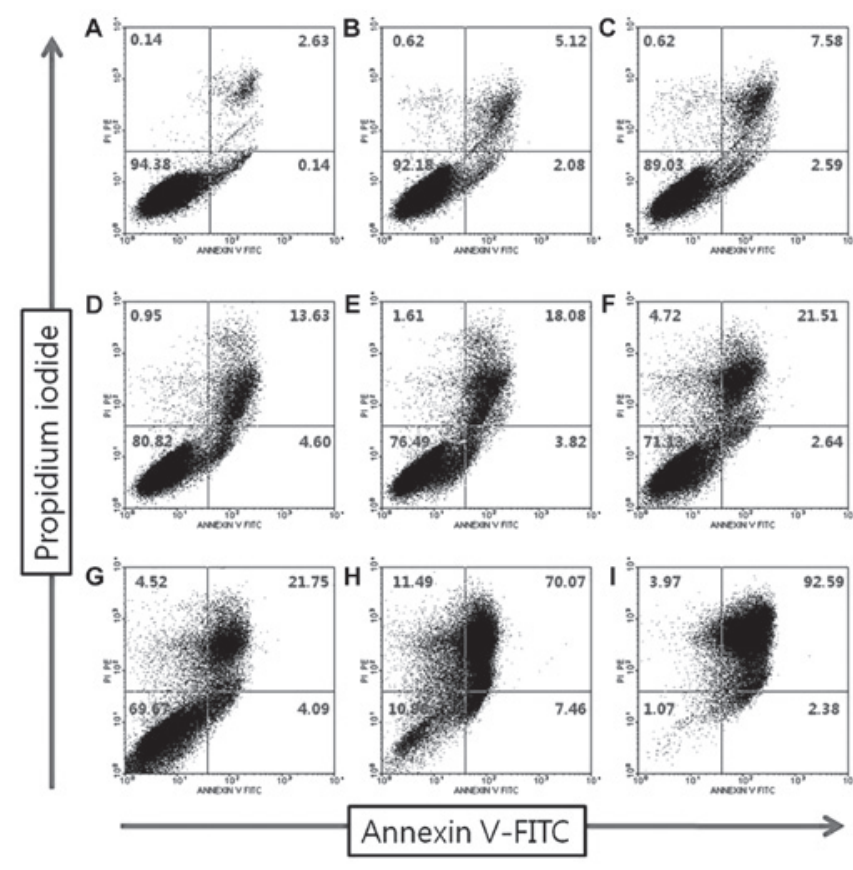

Figure 2. Cells were stained with Annexin V-FITC and PI, and flow cytometry was performed. The percentage of cells in each quadrant is shown. The upper right quadrant shows late apoptosis, and the lower right quadrant early apoptosis. AGS cells were treated with various concentrations of capsaicin for 12 h: (A) Control group; cells were treated with (B) 10, (C) 20, (D) 30 , (E) $60,(\mathrm{~F}) 80,(\mathrm{G}) 100,(\mathrm{H}) 200$ and (I) $300 \mu \mathrm{M}$, respectively. The results are representative of three separate experiments.

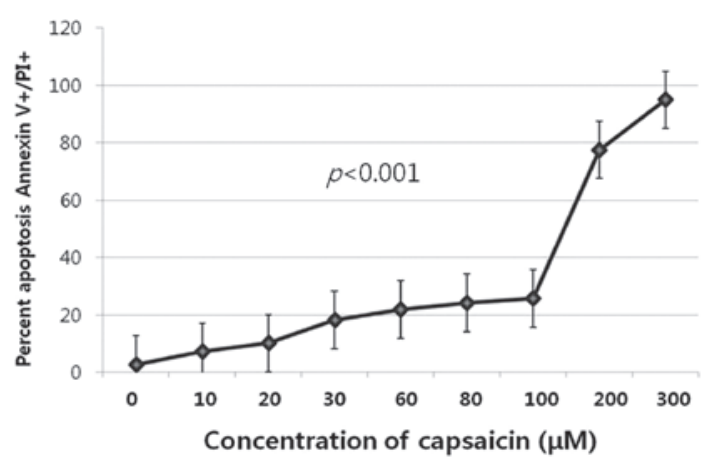

Figure 3. The number of early and late apoptotic cells or directly damaged cells increased after exposure of the cells to different concentration of capsaicin for $12 \mathrm{~h}$. The results are representative of three independent experiments. 


\section{Cont Cap}

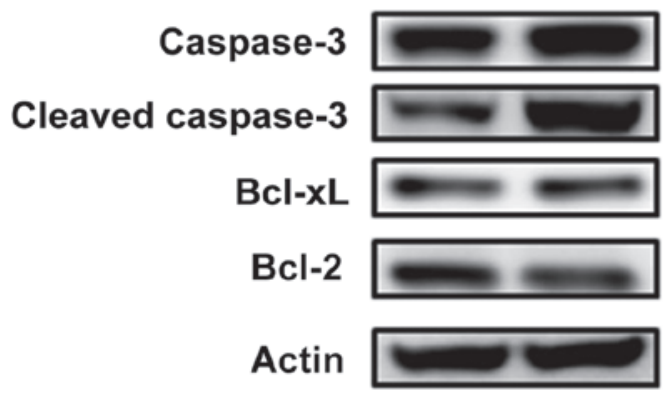

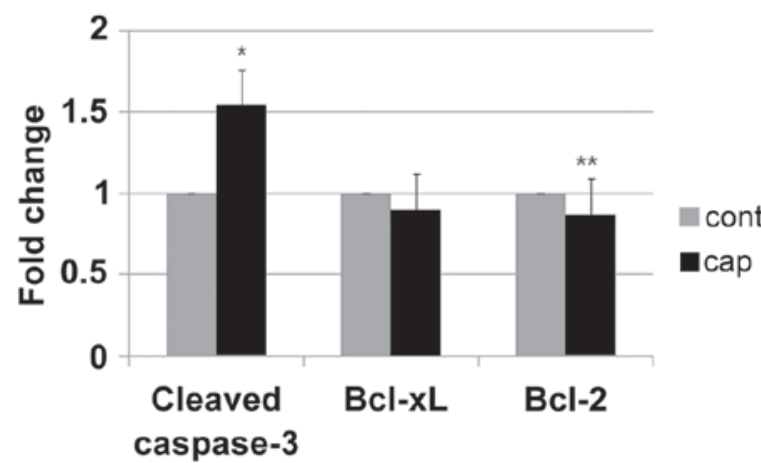

Figure 4. The effects of capsaicin on apoptotic-related protein expression. An increase in cleaved caspase-3 expression was detected in the AGS cells following treatment with capsaicin $(100 \mu \mathrm{M})$ for $12 \mathrm{~h}$. Protein expression of Bcl-2 was reduced in the AGS cells after treatment with capsaicin (100 $\mu \mathrm{M})$ for $12 \mathrm{~h}\left(\right.$ mean $\pm \mathrm{SE},{ }^{*} \mathrm{P}<0.005$ and $\left.{ }^{* *} \mathrm{P}<0.05\right)$.
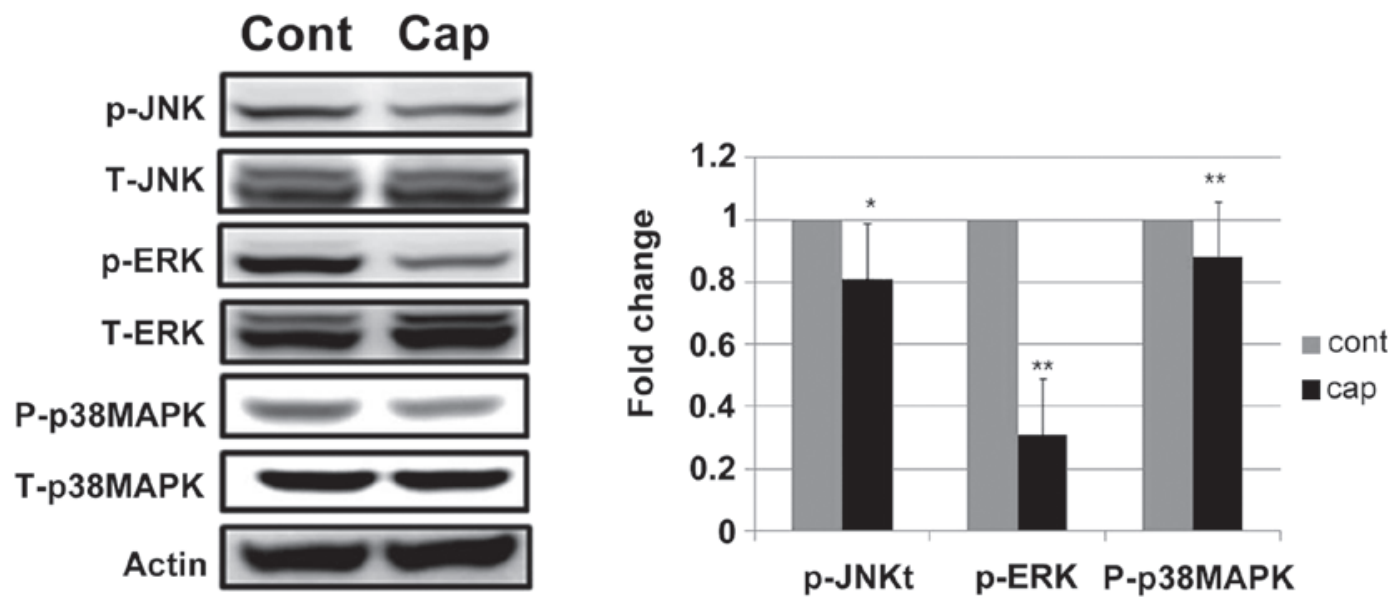

Figure 5. Capsaicin treatment decreased the expression of phosphorylated ERK 1/2, JNK and p38 MAPK. Cells were incubated with or without capsaicin $(100 \mu \mathrm{M})$ for $12 \mathrm{~h}\left(\right.$ mean $\pm \mathrm{SE},{ }^{*} \mathrm{P}<0.005$ and $\left.{ }^{* *} \mathrm{P}<0.05\right)$. The phosphorylation levels of total ERK $1 / 2$, JNK and p38 MAPK were not altered following capsaicin treatment.

is thought to contribute to carcinogenesis $(3,4)$. To evaluate the impact of capsaicin on apoptosis, we performed FACS analysis. Fig. 2 shows flow cytometric plots obtained with Annexin V-FITC/PI assay after a 12-h exposure to different concentrations $(0-300 \mu \mathrm{M})$. The number of early and late apoptotic cells or directly damaged cells increased subsequent to exposure of the cells to capsaicin $(\mathrm{P}<0.001$, Fig. 3$)$.

Capsaicin-induced apoptosis is associated with the modulation of apoptotic regulatory proteins in human gastric cancer cells. An increase in cleaved caspase-3 expression was detected in the AGS cells following treatment with capsaicin (Fig. 4). Apoptosis is regulated by the balance between pro- and anti-apoptotic genes. As shown in Fig. 4, the protein levels of the anti-apoptotic gene Bcl-2 was reduced by treatment with capsaicin in AGS cells, whereas the protein levels of Bcl-xL were not altered in response to the treatment with capsaicin.

Capsaicin modulates the phosphorylation of MAPK cascade signaling proteins in human gastric cancer cells. The effect of capsaicin on the MAPK signal pathway, which is essential for cell survival and growth during development and carcinogenesis, was examined. Capsaicin treatment decreased the expression of phosphorylated ERK 1/2 (Fig. 5). Since crosstalk between signal transduction pathways is common, we examined whether capsaicin prevented the phosphorylation of p38 or JNK in human gastric cancer cells. Capsaicin treatment slightly decreased the phosphorylation of p38 and JNK. The levels of total ERK 1/2, JNK and p38 MAPK were not altered after capsaicin treatment (Fig. 5).

\section{Discussion}

Although previous studies (3-8) have focused on the tumor suppressive effects of capsaicin in human cancer cells, few studies have been undertaken to elucidate capsaicin-induced apoptosis in human gastric cancer cells. Previous results of an XTT assay, DNA fragmentation and flow cytometric analysis revealed that capsaicin (3.5-10 $\mu \mathrm{mol} / \mathrm{l})$ induced apoptotic cell death in an in vitro Korean stomach tumor cell (SNU1) (13). This induction of cell death may be due to a Bcl-2 sensitive apoptotic pathway in a human gastric adenocarcinoma cell line (AGA cells) (12). In this study, cell proliferation significantly decreased in a dose-dependent manner following incubation 
of AGS cells with capsaicin for $12 \mathrm{~h}$. The results have also demonstrated that the proportion of early and late apoptotic cells induced by capsaicin increased in a dose-dependent manner. The anticancer effects of capsaicin are both concentration- and time-dependent in vitro (7). Previous findings have shown the effects of capsaicin to be in the low micromolar range and to become maximal at 200-300 $\mu \mathrm{M}(2)$. Our results have shown that a considerable antiproliferative or apoptotic effect of capsaicin occurred at 60-300 $\mu \mathrm{M}$.

The apoptotic response was evidenced by the activation of caspase-3 generation and the expression of anti-apoptotic markers. The activity of caspase- 3 was found to increase with the exposure to capsaicin. In addition, western blotting revealed a reduction of the $\mathrm{Bcl}-2$ gene. Recently, the $\mathrm{Bcl}-2$ gene appears to be a critical regulator of programmed cell death in a variety of physiological and pathological contexts (14). Additionally, the expression of the Bcl-2 protein was reduced when the AGS cells were exposed to higher doses (10-200 $\mu \mathrm{mol} / \mathrm{l})$ of capsaicin, which was similar to results of the present study (12). These results suggest that capsaicin is potentially involved in the modulation of proliferation and apoptosis in human gastric cancer cells.

The ERK 1/2 pathway has been associated with cell proliferation and differentiation (15). Findings of a recent study have demonstrated that exposure to capsaicin clearly inhibited the activation of ERK in breast cancer cell lines including BT-474 and SK BR-3. In human fibrosarcoma cells, capsaicin suppressed the EGF-induced phosphorylation of EGFR, Akt, Raf, ERK1/2 and p38 MAPK (16), while blocking upstream ERK $1 / 2$ phosphorylation reduced the amount of IL-8 produced by AGS cells in response to $H$. pylori infection (17). In this study, capsaicin suppressed the phosphorylation of ERK 1/2, JNK and p38 MAPK in human gastric cancer cells. Thus, capsaicin may be useful for anti-inflammatory or anti-carcinogenic activity in gastric cancer associated with H. pylori infection.

However, as the pharmacokinetics of capsaicin in human have not yet been investigated, predicting the amount of chili peppers that would need to be consumed to achieve the effective concentration of capsaicin observed is unclear.

Capsaicin reduced cell proliferation and increased apoptosis in a concentration-dependent manner in human gastric cancer cells. Cleaved caspase- 3 was increased and Bcl-2 was reduced by treatment with capsaicin in AGS cells. Capsaicin treatment decreased the expression of phosphorylated ERK 1/2, p38 MAPK or JNK in AGS cells.

In conclusion, results of this study suggest that capsaicin may serve as an anti-tumorigenic agent in human gastric cancer. However, the physiological capsaicin dose for the therapeutic or chemopreventive agents is unclear and remains to be determined in future investigations.

\section{References}

1. Monsereenusorn Y, Kongsamut S and Pezalla PD: Capsaicin - a literature survey. Crit Rev Toxicol 10: 321-339, 1982.

2. Bley K, Boorman G, Mohammad B, McKenzie D and Babbar S: A comprehensive review of the carcinogenic and anticarcinogenic potential of capsaicin. Toxicol Pathol 40: 847-873, 2012.

3. Thoennissen NH, O'Kelly J, Lu D, et al: Capsaicin causes cell-cycle arrest and apoptosis in ER-positive and -negative breast cancer cells by modulating the EGFR/HER-2 pathway. Oncogene 29: 285-296, 2010.

4. Choi CH, Jung YK and Oh SH: Autophagy induction by capsaicin in malignant human breast cells is modulated by $\mathrm{p} 38$ and extracellular signal-regulated mitogen-activated protein kinases and retards cell death by suppressing endoplasmic reticulum stress-mediated apoptosis. Mol Pharmacol 78: 114-125, 2010.

5. Kim YM, Hwang JT, Kwak DW, Lee YK and Park OJ: Involvement of AMPK signaling cascade in capsaicin-induced apoptosis of HT-29 colon cancer cells. Ann NY Acad Sci 1095: 496-503, 2007.

6. Eling TE, Baek SJ, Shim M and Lee CH: NSAID activated gene (NAG-1), a modulator of tumorigenesis. J Biochem Mol Biol 39: 649-655, 2006.

7. Wu CC, Lin JP, Yang JS, et al: Capsaicin induced cell cycle arrest and apoptosis in human esophagus epidermoid carcinoma CE 81T/VGH cells through the elevation of intracellular reactive oxygen species and $\mathrm{Ca}^{2+}$ productions and caspase-3 activation. Mutat Res 601: 71-82, 2006.

8. Chanda S, Erexson G, Frost D, Babbar S, Burlew JA and Bley K: 26-Week dermal oncogenicity study evaluating pure transcapsaicin in Tg.AC hemizygous mice (FBV/N). Int J Toxicol 26: 123-133, 2007.

9. Akagi A, Sano N, Uehara H, Minami T, Otsuka H and Izumi K: Non-carcinogenicity of capsaicinoids in B6C3F1 mice. Food Chem Toxicol 36: 1065-1071, 1998.

10. Wang HM, Chuang SM, Su YC, Li YH and Chueh PJ: Down-regulation of tumor-associated NADH oxidase, tNOX (ENOX2), enhances capsaicin-induced inhibition of gastric cancer cell growth. Cell Biochem Biophys 61: 355-366, 2011.

11. Lee IO, Lee KH, Pyo JH, Kim JH, Choi YJ and Lee YC: Anti-inflammatory effect of capsaicin in Helicobacter pylori-infected gastric epithelial cells. Helicobacter 12: 510-517, 2007.

12. Lo YC, Yang YC, Wu IC, et al: Capsaicin-induced cell death in a human gastric adenocarcinoma cell line. World $\mathrm{J}$ Gastroenterol 11: 6254-6257, 2005.

13. Kim JD, Kim JM, Pyo JO, et al: Capsaicin can alter the expression of tumor forming-related genes which might be followed by induction of apoptosis of a Korean stomach cancer cell line, SNU-1. Cancer Lett 120: 235-241, 1997.

14. Weil M, Jacobson MD, Coles HS, et al: Constitutive expression of the machinery for programmed cell death. J Cell Biol 133: 1053-1059, 1996.

15. Schlessinger J: Cell signaling by receptor tyrosine kinases. Cell 103: 211-225, 2000.

16. Hwang YP, Yun HJ, Choi JH, et al: Suppression of EGF-induced tumor cell migration and matrix metalloproteinase-9 expression by capsaicin via the inhibition of EGFR-mediated FAK/Akt, PKC/Raf/ERK, p38 MAPK, and AP-1 signaling. Mol Nutr Food Res 55: 594-605, 2011.

17. Keates S, Keates AC, Warny M, Peek RM Jr, Murray PG and Kelly CP: Differential activation of mitogen-activated protein kinases in AGS gastric epithelial cells by $\mathrm{cag}^{+}$and cag Helicobacter pylori. J Immunol 163: 5552-5559, 1999. 